\title{
Ordinal Magnitude Scaling for Automated Lane Keeping Displays
}

\author{
Brittany E. Noah \\ Georgia Institute of Technology \\ Atlanta, GA 30306, USA \\ brittany.noah@gatech.edu \\ Thomas M. Gable \\ Georgia Institute of Technology \\ Atlanta, GA 30306, USA \\ thomas.gable@gatech.edu \\ Bruce N. Walker \\ Georgia Institute of Technology \\ Atlanta, GA 30306, USA \\ bruce.walker@gatech.edu
}

Permission to make digital or hard copies of part or all of this work for personal or classroom use is granted without fee provided that copies are not made or distributed for profit or commercial advantage and that copies bear this notice and the full citation on the first page. Copyrights for third-party components of this work must be honored. For all other uses, contact the Owner/Author.

uses, contact the Owner/Author.
Copyright is held by the owner/author(s).

Automotive'UI 16 Adjunct, October 24-26, 2016, Ann Arbor, MI, USA ACM $978-1-4503-4654-2 / 16 / 10$

http://dx.doi.org/10.1145/3004323.3004341

\begin{abstract}
It is crucial for drivers to be able to understand the state and reliability of vehicle automated systems.

Visual displays may be helpful in conveying automation status, but they must be designed carefully, with full understanding of drivers' mental models, and be evaluated to verify the display's communicative intent is being realized. The current paper presents and tests basic visual dynamic displays to display this information and discusses a methodology to test user mental models. Participants viewed 9 possible display manipulations in the context of lane keeping, each with 5 levels of information to determine matches with users' expectations. The results point to mixed mental models regarding the displays, showing the importance of empirical testing with users. The methods and findings are discussed in terms of application to the future design of "human-automation interfaces".
\end{abstract}

\section{Author Keywords}

Autonomous vehicle; automation displays; reliability display; required driver engagement.

\section{ACM Classification Keywords}

H.1.2 [User/Machine Systems]: Human factors; H.5.2 [Information Interfaces And Presentation (e.g., HCI)]: Evaluation/methodology, Graphical user 
interfaces (GUI), Screen design (e.g., text, graphics, color), User-centered design.

\section{Introduction}

The safety of semi-autonomous vehicles has recently been prevalent in the news with a number of deaths occurring in semi-autonomous vehicles while the automation was engaged $[6,9]$. Another news story was recently published that critiqued the clarity of information regarding the vehicle state of a new vehicle with some semi-autonomous safety features [1]. These stories show the real life examples of what can happen when drivers have poor awareness of their vehicle's automation status. This is an issue that is vital to document, understand, and correct as we move towards a more automated future, with vehicles performing more of the driving maneuvers and operators becoming more automation supervisors.

Automation supervision

Humans are not inherently good at supervising technologies [5]. Even with training, humans are not the ideal supervisors of systems and can make deadly errors [2]. An important part of the supervision task within an automated system is having situation awareness (SA). SA is defined as users understanding the system and the system's context in the operational environment. There are three levels of SA defined by Endsley [4]: perception, understanding, and projection. Perception (level 1 ) is the first stage of SA, and indicates that users are able to perceive the system status and environment. Understanding (level 2) is a moderate level of SA, indicating that users are able to turn the perceptions into a meaningful understanding of the system. The ability to predict future system states (level 3 ) is the highest level of SA. Predicting future states of the vehicle, its environment, and the automation is particularly important in driving (supervising) cars with automated processes. Trend displays are posited as ways to give users information over time to assist in such predictive abilities [4].

\section{Automation displays}

Current semi-autonomous vehicles do not give drivers insight into how the automation makes decisions or the reliability of the automation. This lack of transparency about the automation has already been shown to have deadly consequences. Instead, the systems require the driver to continually watch the road and what the vehicle is doing to ensure a system match. The creation of a display that could be used specifically to inform the driver of the status (confidence/reliability) of the vehicle's automation would be helpful with level 3 SA. This could help drivers know when they need to be more alert/aware/engaged as they supervise their car.

\section{Automation Reliability}

Reliability of automation is defined as performance of the automated system as measured by the number of errors made by a system during a given amount of time. At high levels of automation reliability (corresponding to fewer automation errors), intermittent failures may not have an impact on trust in the system unless the failures continue to occur over time, manifesting themselves at critical points of operation [5, 8]. In aviation, there are many examples of failed warning systems and over-reliance on automated features of flight that, when undetected, lead to accidents [8]. Error detection has been shown to be an important factor in overall human-automation performance for supervisory control tasks [7]. As more cars with automated features are being developed, 
displays will become even more critical to ensuring drivers understand how to use the technology in their vehicle, and when to intervene if it fails.

RELIABILITY DISPLAYS

To display automation status to drivers, a mental model, or representation of the information, has to be determined. There are two possible metrics that can be used to display this information to drivers. The automation reliability (AR), a system-centric measure, can be displayed to drivers. Alternatively, the required driver engagement (RDE) can be displayed, which tells the driver directly when their attention is more necessary. RDE is a more human-centric measure. These two measures of automation reliability were chosen to determine whether participants found one metric to be easier to understand than the other. In addition, preference for one metric over another could have implications in further behavioral studies. Determining which display type would be most intuitive and most preferable for drivers to act upon in response to a decrease in automation reliability and/or a failure of the automated system is therefore vital to safety.

To ensure displays are well designed, we must determine how the displays correspond to participants' mental models of high AR/low RDE and low AR/high RDE. Displays that show variable results may confuse potential users. If displays can represent high RDE/low AR mappings to users, then these will be more intuitive, regardless of whether companies decide to design the display in a AR or an RDE approach.

Present study

The present study serves two purposes, to present the findings of an investigation into the mental models for possible users of different types of dynamic displays on how they match to AR and RDE, and, to present a method for testing mental models for such displays. The first is being done to ensure designers have some guidance for automation displays in vehicles. The second is to give designers and researchers tools for assessing displays in the future. The method used here is based on magnitude estimation-often used in research looking at how people perceive sound [10]. Magnitude estimation offers participants more freedom to assign meaning to the sounds or, in this case, images that they are presented with. In this study, participants sorted the circle stimuli into five different levels along the dimensions of AR and RDE. This method of estimating magnitude in discrete categories is called ordinal magnitude estimation.

\section{Methods}

\section{Participants}

Thirty-six students ( 21 male, 14 female, and 1 person who chose not to identify their gender) participated in this study. They were on average 19.6 years old $(\mathrm{SD}=1.5)$

\section{Materials}

The forced choice sorting task was conducted in a lab using a computer program developed for this purpose.

\section{Design Considerations}

To determine what types of image manipulations were associated with high and low levels of AR and RDE, sets of circles varied on only one of the following dimensions: horizontal fill, vertical fill, radial fill, ovement, flash rate, opacity, size, dashed/solid outline, and color. Five circles were presented within each display manipulation (see Table 1 ). 


\begin{tabular}{|c|c|c|c|c|c|}
\hline Dimension & 1 & 2 & 3 & 4 & 5 \\
\hline \multicolumn{6}{|l|}{ Dashes } \\
\hline Flashing & $10 \mathrm{~Hz}$ & $2 \mathrm{~Hz}$ & $1 \mathrm{~Hz}$ & $0.667 \mathrm{~Hz}$ & $0.5 \mathrm{H}$ \\
\hline \multicolumn{6}{|l|}{$\begin{array}{l}\text { Horizontal } \\
\text { Fill }\end{array}$} \\
\hline \multicolumn{6}{|l|}{$\begin{array}{l}\text { Vertical } \\
\text { Fill }\end{array}$} \\
\hline \multicolumn{6}{|l|}{ Radial fill } \\
\hline \multicolumn{6}{|l|}{ Opacity } \\
\hline \multicolumn{6}{|l|}{ Movement } \\
\hline \multicolumn{6}{|l|}{ Size } \\
\hline Color & 0 & . & 0 & 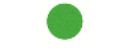 & \\
\hline
\end{tabular}

\section{Procedure}

Participants viewed each circle type in random order and sorted the circles in that set into levels of high to low $A R$, and then for RDE (counterb 'alanced). An example of the sorting task can be seen in Figure 1.

\begin{abstract}
Results
$A R$

For $\boldsymbol{A R}$ (see Table 2), the mode of the ratings for the circle dimensions followed a pattern of increasing from least to greatest on each dimension. For example, as the dashed circle became more solid, AR increased; similarly, as the horizontal fill circles became fuller AR increased. The only manipulation that did not follow this pattern was opacity: highly opaque circles were mapped to low AR and highly transparent circles mapped to high AR.
\end{abstract}

\section{$R D E$}

For $\boldsymbol{R D E}$ (see Table 3), the modes were opposite of the pattern seen in AR for some manipulations and remained the same in others. For example, the circle with the most dashes corresponded to the highest level of RDE. The same was true for the flashing circles: as the rate of flashing increased, so too did the level of RDE. The color manipulation was also opposite of the $A R$ results: as the circles went towards red the RDE increased. All other display manipulations had modes across levels that suggested participants felt the same way as in the AR sorting task.

\section{Discussion}

In the present study, participants sorted 5 dimensions for 9 types of display manipulations in regards to how they corresponded to high/low automation AR and RDE. Four of the dynamic displays were mapped to have high RDE and low $A R$, meaning that these display manipulations correspond to action required by the driver across both mental models or display design types. High flash rates, high opacity, more solid circles, and red color as opposed to green were all characteristics of the manipulations that were chosen 


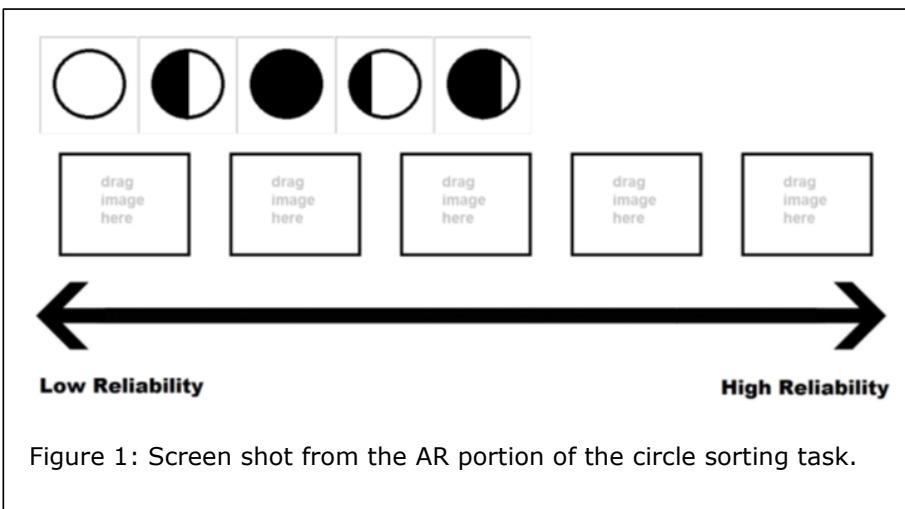

most frequently for these low levels of AR and high RDE and vice versa. This means that these 4 design types are seen in the same way across the participants and make them good candidates for AR/RDE displays in the vehicle as they will not be as easily mixed up in users' mental models or across different display approaches. While only simple circle

\begin{tabular}{|c|c|c|c|c|c|c|}
\hline \multicolumn{9}{|c|}{ AR } \\
\hline \multirow{2}{*}{ Dimension } & & Lowest & Low & Moderate & High & Highest \\
\hline \multirow{2}{*}{ Dashed } & Mode & 1 & 2 & 3 & 4 & 5 \\
\cline { 2 - 7 } & Variance & 0.863 & 0.257 & 0.085 & 0.237 & 0.863 \\
\hline \multirow{2}{*}{ Flashing } & Mode & 1 & 2 & 3 & 4 & 5 \\
\cline { 2 - 8 } & Variance & .340 & 0.743 & 0.180 & 0.692 & 1.99 \\
\hline \multirow{2}{*}{ Horizontal Fill } & Mode & 1 & 2 & 3 & 4 & 5 \\
\cline { 2 - 8 } & Variance & 3.679 & 0.914 & 0.142 & 0.971 & 3.616 \\
\hline \multirow{2}{*}{ Vertical Fill } & Mode & 1 & 2 & 3 & 4 & 5 \\
\cline { 2 - 8 } & Variance & 3.679 & 0.914 & 0.142 & 0.971 & 3.616 \\
\hline \multirow{2}{*}{ Radial fill } & Mode & 1 & 2 & 3 & 4 & 5 \\
\cline { 2 - 8 } & Variance & 3.492 & 0.914 & 0.000 & 0.936 & 3.533 \\
\hline \multirow{2}{*}{ Movement } & Mode & 1 & 2 & 3 & 4 & 5 \\
\cline { 2 - 8 } & Variance & 2.016 & 2.149 & 0.421 & 0.828 & 2.057 \\
\hline \multirow{2}{*}{ Opacity } & Mode & 5 & 4 & 3 & 2 & 1 \\
\cline { 2 - 7 } & Variance & 4.102 & 1.025 & 0.000 & 1.025 & 4.102 \\
\hline \multirow{2}{*}{ Size } & Mode & 1 & 2 & 3 & 4 & 5 \\
\cline { 2 - 7 } & Variance & 3.657 & 0.914 & 0.000 & 1.018 & 3.533 \\
\hline \multirow{2}{*}{ Color } & Mode & 1 & 2 & 3 & 4 & 5 \\
\cline { 2 - 7 } & Variance & 1.111 & 0.733 & 0.307 & 0.686 & 1.6 \\
\hline
\end{tabular}

Table 2: AR ratings for the 5 dimensions of each type of circle. displays were used here, these mappings should be expected to carry over to other types of

shapes/displays. The other display dimensions may not lead to consistent interpretations, and thus should be avoided, or at least used with great caution.

The method used here was effective for showing how mental models of the participants matched to the display manipulations presented. The modes of each metric level were an effective method of sorting the data and variance across participants, and showed the level of agreement for each manipulation and dimension. This variance could be used in further work to determine the best overall candidate, which here, seemed to be the flashing displays due to the low variance across the levels of both RDE and AR.

Further research should be done in this space to determine the most ideal mappings for in-vehicle situations with an automated system in place.

Behavioral studies determining which displays are most easily acted upon by drivers will give more insight into whether AR or RDE displays would be most effective in the automated driving context. These initial non-driving results give a general idea of potential user mental mappings. While this paper gives a few options for how the two design approaches of AR or RDE displays should be matched, it may be safer and more effective to use the displays employing dashed, flashing, opacity, and color whose mappings were agreed upon across the two factors. The other display types of horizontal fill, vertical fill, radial fill, movement, and size should be used carefully as it could create a cross-over in users' mental models and cause confusion of driver responsibilities. 


\begin{tabular}{|c|c|c|c|c|c|c|}
\hline \multicolumn{7}{|c|}{ RDE } \\
\hline Dimension & & Lowest & Low & Moderate & High & Highest \\
\hline \multirow{2}{*}{ Dashed } & Mode & 5 & 4 & 3 & 2 & 1 \\
\hline & Variance & 3.086 & 0.828 & 0.114 & 0.714 & 3.056 \\
\hline \multirow{2}{*}{ Flashing } & Mode & 5 & 4 & 3 & 2 & 1 \\
\hline & Variance & 0.993 & 0.454 & 0.307 & 0.136 & 1.075 \\
\hline \multirow{2}{*}{ Horizontal Fill } & Mode & 1 & 2 & 3 & 4 & 5 \\
\hline & Variance & 2.844 & 0.711 & 0.028 & 0.713 & 2.844 \\
\hline \multirow{2}{*}{ Vertical Fill } & Mode & 1 & 2 & 3 & 4 & 5 \\
\hline & Variance & 2.286 & 0.943 & 0.000 & 0.644 & 2.406 \\
\hline \multirow{2}{*}{ Radial fill } & Mode & 1 & 2 & 3 & 4 & 5 \\
\hline & Variance & 2.286 & 0.571 & 0.000 & 0.571 & 2.286 \\
\hline \multirow{2}{*}{ Movement } & Mode & 1 & 2 & 3 & 4 & 5 \\
\hline & Variance & 2.600 & 1.044 & 0.421 & 1.771 & 3.190 \\
\hline \multirow{2}{*}{ Opacity } & Mode & 1 & 2 & 3 & 4 & 5 \\
\hline & Variance & 1.968 & 0.492 & 0.00 & 0.492 & 1.968 \\
\hline \multirow{2}{*}{ Size } & Mode & 1 & 2 & 3 & 4 & 5 \\
\hline & Variance & 1.968 & 0.492 & 0.000 & 0.618 & 1.799 \\
\hline \multirow{2}{*}{ Color } & Mode & 5 & 4 & 3 & 2 & 1 \\
\hline & Variance & 1.111 & 0.733 & 0.307 & 0.686 & 1.600 \\
\hline
\end{tabular}

\section{Acknowledgements}

The authors would like to thank Shruti Singh, Shao-Yu Chen, Woody Shortridge, and Brianna Tomlinson for their assistance in developing the stimuli and processing the data for this study. Portions of the work were supported by a National Science Foundation Graduate Research Fellowship (DGE-1148903).

\section{References}

1. Adams, E (2016, June 27). Mercedes's New E-Class Kinda Drives Itself-And It's Kinda Confusing. Wired. https://www.wired.com/2016/06/mercedess-newe-class-kinda-drives-kinda-confusing/

2. Cooke, N. J. \& Durso, F. (2008). Stories of Modern Technology Failures and Cognitive Engineering Successes. Boca Raton, FL: CRC Press.

3. Endlsey, M. R. (1995). Toward a theory of situation awareness in dynamic systems. Human Factors 37(1) 32-64.

4. Endsley, M. R. (1998). Design and evaluation for situation awareness enhancement. Proceedings of the Human Factors Society $32^{\text {nd }}$ Annual Meeting. Santa Monica, CA: Human Factors Society. pp. 97101

5. Parasuraman, R. (1997). Humans and Automation Use, Misuse, Disuse, Abuse. Human Factors 39(2), 250-253.

6. Singhvi, A. \& Russel, K. (2016, July 12). Inside the Self-Driving Tesla Fatal Accident. The New York Times.

http://www.nytimes.com/interactive/2016/07/01/b usiness/inside-tesla-accident.html

7. Sheridan, T. B. (2012). Human Supervisory Control. In G. Salvendy (Ed.). Handbook of Human Factors and Ergonomics (p. 990-1011). Hoboken, NJ: Wiley and Sons.

8. Stanton, N. A. \& Marsden, P. (1996). From Fly-ByWire to Drive-By-Wire: Safety implications of automation in vehicles. Safety Science 24(1), 3549.

9. Vlasic, B. \& Boudette, N. E. (2016, June 30). SelfDriving Tesla Was Involved in Fatal Crash, U.S. Says. The New York Times. http://www.nytimes.com/2016/07/01/business/self -driving-tesla-fatal-crash-investigation.html?_r=0

10. Walker, B. N. (2002). Magnitude estimation of conceptual data dimensions for use in sonification. Journal of Experimental Psychology: Applied 8(4), 211-221. 